\title{
Addressing donor-organ shortages using extended criteria in lung transplantation
}

\author{
Hannah Neizer, Gaurev B. Singh, Shyla Gupta, Steve K. Singh \\ Trillium Health Partners, University of Toronto, Toronto, Canada \\ Correspondence to: Steve K. Singh, MD, MSc. Trillium Health Partners, University of Toronto, Toronto, Canada. Email: steve.singh@thp.ca.
}

Submitted Sep 02, 2019. Accepted for publication Oct 11, 2019.

doi: 10.21037/acs.2019.10.01

View this article at: http://dx.doi.org/10.21037/acs.2019.10.01

Lung transplantation is an established therapy for patients with end-stage pulmonary disease. Over the last few decades, advancements in surgical techniques, post-operative care and immunosuppression therapy have led to improved survival outcomes following lung transplantation; however, pre-transplant waiting lists continue to increase in number, with an increasing number of listed recipients requiring hospital admission, often for mechanical ventilator support and/or extra-corporeal membrane oxygenation (ECMO) support prior to transplant. In North America, over twothousand (approximately 15\%) adult patients listed for lung transplant either die or are removed from the wait list, for becoming too sick to transplant (1).

The shortage of donor organs remains a major limitation for transplantation. Currently, the 'ideal' lung donor is said to be someone between the ages of 20 to 45 , has an arterial partial pressure of oxygen $\left(\mathrm{PaO}_{2}\right) / \mathrm{FiO}_{2}$ ratio greater than 350 , has no smoking history, a clear chest $\mathrm{X}$-ray, a clean bronchoscopy and minimal ischemic time ( $<6-8$ hours) (2). The quality of the donor's lungs has an undisputedly significant impact on both early and long-term outcomes after transplantation. However, the empiric definition set in the 1980s results in utilization of $<30 \%$ of available donor lungs (1). In order to increase the supply of donor lungs, the idea of extending donor criteria is being heavily discussed within the medical community and employed in the contemporary practice of major programs aiming to treat their growing list of patients.

Extended donor criteria (EDC) refers to the use of donor lungs that do not meet the standard criteria for transplantation (2). Organ procurement organizations and transplant centers are often reluctant to use 'marginal' donor organs that do not meet the established guidelines due to the inherent risk and lack of research. However, the first clarification we must make are that extended donor criteria does not imply marginal or sub-standard quality. Contemporary extension of donor variables has been based on evidence of both acceptable early and late graft survival and function. Evidence suggests that older donors, history of smoking or asthma and/or cause of death (i.e., drowning or hanging), should not be considered absolute contraindications when evaluated, if radiographic imaging and $\mathrm{P} / \mathrm{F}$ ratios are $>300$ and maintain a positive trend $(3,4)$. Although matching virology such as Epstein-Barr Virus (EBV) has been shown to impact outcomes, evidence has shown that managing donors with hepatitis $\mathrm{C}$ (antigen positive or negative) with novel hepatitis $\mathrm{C}$ treatment, posttransplant in to non-hepatitis $\mathrm{C}$ recipients, does not impact early clinical outcomes (5). In the current era, where we face an opioid crisis and increasing intravenous drug abuse, which sadly leads to premature deaths, there is solace in the potential use of these organs for transplant.

Medium-to-high volume lung transplantation programs are now using ex-vivo lung perfusion (EVLP) as an increasingly viable option that can increase the donor pool in two ways $(6,7)$. Firstly, EVLP aids in reconditioning less desirable lungs via proprietary perfusate, which prevents further injury and improves lung physiology, therefore salvaging lungs that do not meet the ideal $\mathrm{PaO}_{2}$ criteria through optimization of arterial partial pressure prior to transplantation. Secondly, this approach not only resuscitates lungs that were inappropriate for transplant, but in acceptable lungs, it also increases the procurement distance/time-frame. Studies suggest that EVLP may remove the time limitations imposed by cold ischemia, reducing the risk of viable donor lungs that would otherwise be discarded.

A key factor often ignored in the debate regarding using 
extended donor criteria, is patient autonomy. This refers to the moral right of an individual to follow and make free choices related to his or her life plan. Determined by the lung allocation score (LAS), priority is given to patients with the least amount of time to live and those who are most likely to survive the operation, in order to minimize futile transplants. If patients are low on the LAS score, are they willing to take an extended criteria donor (ECD) lung to improve their odds of receiving a transplant? There is currently limited research with respect to ECD lungs; however, a 2011 study evaluated the willingness of patients to accept ECD livers. This study suggested that patients who are at higher risk of mortality are more willing to accept a transplant from a marginal donor (8). In addition, another study indicated that a large component of patients' willingness to accept an ECD organ was based on how the information was communicated, their current health status and the potential health benefits to the recipient (9). In spite of these findings, more research needs to be conducted specifically in regards to how the accepting physicians communicate the decision-making regarding extended criteria donors accepted to patients and research on the spectrum of recipients' willingness to accept these organs, whether it is uniform in their values, or dependent on their clinical situation at the time of offer. Ultimately, they should be involved in the decision during the consent process.

The global shortage of donor lungs for transplantation is recognized as a major public health challenge with aims to increase public awareness and discussion surrounding organ donation. The lung transplant community continues to drive the redefinition of donor lung criteria with outcomesbased research. Technologies such as EVLP and organ perfusion transport systems aim to both resuscitate organs and preserve them over longer distances. Patients must remain central in the informed-consent process of utilizing these organs. All these strategies can continue the trend of increasing donor organs, ultimately averting the risks that persist as patients remain on transplant waiting lists.

Cite this article as: Neizer H, Singh GB, Gupta S, Singh SK. Addressing donor-organ shortages using extended criteria in lung transplantation. Ann Cardiothorac Surg 2020;9(1):49-50. doi: $10.21037 /$ acs.2019.10.01

\section{Acknowledgments}

None.

\section{Footnote}

Conflicts of Interest: The authors have no conflicts of interest to declare.

\section{References}

1. Keeshan BC, Rossano JW, Beck N, et al. Lung transplant waitlist mortality: height as a predictor of poor outcomes. Pediatr Transplant 2015;19:294-300.

2. Chaney J, Suzuki Y, Cantu E 3rd, et al. Lung donor selection criteria. J Thorac Dis 2014;6:1032-8.

3. Aigner C, Winkler G, Jaksch P, et al. Extended donor criteria for lung transplantation - a clinical reality. Eur J Cardiothorac Surg 2005;27:757-61.

4. Hoang V, Medrek SK, Pendurthi M, et al. Improving Donor Lung Management and Recipient Selection in Lung Transplantation. Am J Respir Crit Care Med 2017;196:782-4.

5. Woolley AE, Singh SK, Goldberg HJ, et al. Heart and lung transplants from HCV-infected donors to uninfected recipients. N Engl J Med 2019;380:1606-17.

6. Cypel M, Yeung JC, Liu M, et al. Normothermic ex vivo lung perfusion in clinical lung transplant. N Engl J Med 2011;364:1431-40.

7. Pan X, Yang J, Fu S, et al. Application of ex vivo lung perfusion (EVLP) in lung transplantation. J Thorac Dis 2018;10:4637-42.

8. Volk ML, Tocco RS, Pelletier SJ, et al. Patient decision making about organ quality in liver transplantation. Liver Transpl 2011;17:1387-93.

9. Rodrigue JR, Hanto DW, Curry MP. Patients' Willingness to Accept Expanded Criteria Donor Liver Transplantation. Am J Transplant 2011;11:1705-11. 